\title{
Correlação entre estabilização central do tronco e valgo dinâmico do joelho em atletas de futsal e futebol
}

http://dx.doi.org/10.11606/1807-5509201900040561

\author{
Camile Ludovico ZAMBOTI* \\ Luana Pezarini MAZZER* \\ Ariobaldo FRISELLI* \\ Jayne Maria BORIN** \\ Christiane de Souza Guerino MACEDO*
}

*Universidade Estadual de Londrina, Londrina, PR, Brasil. **Universidade Norte do Paraná, Londrina, PR, Brasil.

\section{Resumo}

Acredita-se que o aumento de estabilidade e controle neuromuscular do complexo lombo-pélvico-quadril diminui o risco de lesões no joelho, entretanto os resultados de estudos atuais apresentam divergências. 0 objetivo deste estudo é averiguar a correlação entre estabilização central e valgo dinâmico do joelho em atletas. A amostra total foi composta de 20 atletas ( 6 de futsal feminino e 14 de futebol masculino), que foram aleatorizados para a sequência de realização dos testes. Todos foram submetidos ao Bridge Prone Test (BPT) e Step Down Test (SDT), para análise da estabilidade central e valgo dinâmico do joelho, respectivamente. No BPT foram orientados a sustentar a posição de prancha em prono pelo maior tempo possivel, três repetições com descanso de um minuto entre elas, considerado o tempo em segundos. Para o SDT os atletas foram posicionados sobre a plataforma de 20 centímetros, com três marcadores reflexivos e realizaram 10 movimentos de flexão do joelho em apoio unipodal, considerou-se a posição do joelho em apoio para a análise. O SDT foi filmado e analisado pelos softwares Virtual Dub ${ }^{\circledR}$ e Image ${ }^{\circledR}$, e para análise estatística utilizou-se os testes Shapiro Wilk, Mann Whitney U e teste de Correlação de Spearmann, com nível de significância de 5\%, por meio do SPSS 20. Obteve-se como resultados, diferença quando comparado o desempenho em prancha ventral entre o grupo feminino e masculino $(p=0,028)$. Entretanto, quando considerado a prancha e o valgo dinâmico apresentou-se fraca correlação $(r=0,109)$, como também quando separados sexo masculino $(r=0,279)$ e feminino $(r=0,198)$. Conclui-se que houve fraca correlação entre a estabilização do tronco e o valgo dinâmico no joelho nos atletas avaliados, porém observou-se diferença a favor do grupo masculino no BPT.

Palavras-Chave: Fisioterapia; Atletas; Estabilização; Valgo do Joelho.

\section{Introdução}

O futebol e o futsal atraem cada vez mais adeptos, e são considerados esportes de fácil acesso para toda população. Entretanto, em função do grande número de adeptos, da intensidade e frequência de treinamentos apresentam alta incidência de lesões, fator que estimula o interesse dos profissionais da saúde ${ }^{1}$. O futebol é responsável por grandes índices de lesôes dentro das modalidades esportivas de $50-60 \%{ }^{2}$, que podem acometer as mais diversas articulaçóes do corpo, como principal mecanismo de lesóes apontamse os fatores indiretos, independente do contato físico entre os jogadores ${ }^{3}$. Segundo Heidt, et al. ${ }^{4} 58-88 \%$ dessas lesóes acometem as articulaçóes do joelho e tornozelo; onde as lesóes do joelho são mais graves ${ }^{5}$.

O futebol de salão (futsal), por ter características semelhantes às do futebol de campo, tem conquistado um grande número de participantes. Com a sua imensa proliferação, o espaço do futsal realmente se consolidou e adquiriu importância destacada junto à população ${ }^{6,7}$. A evolução da prática do futsal está associada basicamente aos aspectos táticos, técnicos e físicos, exigindo, em consequência disto, cada vez melhor performance do atleta. Desta forma, gera sobrecarga excessiva de treinamento que pode 
desencadear a curto, médio ou longo prazo, traumas de diferentes graus no aparelho musculoesquelético, aumentando o risco para lesōes ${ }^{8,9}$.

Com enfoque aos fatores indiretos, várias alterações cinéticas e cinemáticas nas articulações proximais e distais dos membros inferiores (MMII) podem predispor o atleta a apresentar lesóes no joelho durante a realização de suas atividades esportivas ${ }^{10-12}$. Acredita-se que a estabilidade dinâmica do joelho depende das informações sensoriais acuradas seguidas de uma resposta motora rápida e apropriadas, a qual irá provocar rápidas mudanças de posição de tronco ${ }^{10}$. Um dos fatores que pode contribuir para as lesões desta articulação é a incapacidade do atleta em manter um bom alinhamento entre os segmentos corporais dos membros inferiores, no plano frontal e transverso durante a pratica esportiva ${ }^{3,13}$, como exemplo o valgo dinâmico do joelho.

A avaliação do valgo dinâmico do joelho por imagem é amplamente utilizada no esporte já que alterações dinâmicas desta articulação associadas às atividades complexas dos gestos esportivos podem aumentar a demanda imposta sobre o sistema músculo esquelético, e favorecer o desenvolvimento de vários tipos de lesōes e disfunções nessa articulação ${ }^{14}$, com prejuízo ao atleta e sua equipe. $\mathrm{O}$ valgo dinâmico do joelho pode ser definido como o resultado do aumento da rotação interna, adução do fêmur e da pronação do pé ${ }^{15}$, de modo que durante a adução do quadril, o fêmur roda internamente e o joelho é colocado em posição de valgismo ${ }^{16}$. Essa alteração de alinhamento dinâmico impõe forças rotacionais e de cisalhamento na articulação do joelho, com aumento da sobrecarga do ligamento cruzado anterior (LCA). Como também, pode alterar dinamicamente o alinhamento da patela, e aumentar a sobrecarga em estruturas como os retináculos patelares, cartilagem articular e coxim adiposo, e predispor o desenvolvimento de dor anterior no joelho ${ }^{17} \mathrm{e}$ tendinopatias ${ }^{18}$.

Adicionado a esses fatores, HALL ${ }^{19}$ consideram que o quadril e tronco influenciam de maneira indireta na cinemática do joelho, assim o aumento de estabilidade e controle neuromuscular do complexo lombo-pélvico-quadril diminui o risco de lesões no joelho, principalmente em mulheres ${ }^{20}$, ainda HEWETT, et al. ${ }^{21}$ complementa que o deslocamento do tronco prediz risco de lesões do joelho. Por fim, em recente revisão sistemática CASHMAN ${ }^{22}$ cita que o nível de evidência da relação entre os estabilizadores de quadril e o ângulo de projeção do plano frontal (APPF) é fraco e ainda não é possível alcançar conclusões definitivas.

Assim, devido associação da grande incidência de lesões de joelho com o posicionamento em valgo ${ }^{23,24}$, o presente estudo tem por objetivo correlacionar a estabilidade do complexo lombo-pélvico-quadril com o valgo dinâmico do joelho em atletas, por meio de testes funcionais. Bem como avaliar o valgo dinâmico do joelho do atleta (Step Down Test) e a capacidade de resistência dos músculos tônicos do CORE (Bridge Prone Test) em função do gênero.

\section{Método}

A pesquisa foi conduzida de acordo com a Resolução 466/2012, do Conselho Nacional de Saúde do Ministério da Saúde - Brasília/DF, e aprovada pelo comitê de ética local (parecer 1.025.877). Este estudo se caracteriza como transversal, na área de fisioterapia, exercício físico, controle motor e biomecânica.

Como critérios de inclusão foram estabelecidos aos voluntários serem atletas de futebol ou futsal, com treinamentos diários e participação em competiçôes estaduais e nacionais. Os critérios de exclusão incluíram: queixa álgica de dor em membros inferiores, história de cirurgia no membro inferior nos últimos seis meses ${ }^{25}$. De acordo com o relato da literatura atual sobre a associação de lesões com o valgo do joelho ${ }^{23}$ e para maior objetividade do estudo foram excluídos os indivíduos que realizaram desempenho em varo do joelho durante realização do Step Down Test (SDT).

Inicialmente foram avaliados 29 atletas, 11 de futsal feminino e 18 de futebol masculino. Após a avaliação, foram excluídos nove atletas por apresentarem varo do joelho durante a execução do SDT. A amostra foi de conveniência, composta por 20 atletas, seis atletas do futsal feminino e 14 de futebol masculino. Os atletas foram esclarecidos sobre o procedimento, preencheram o questionário de dados pessoais e assinaram o Termo de Consentimento Livre e Esclarecido. Na sequência foram encaminhados para o protocolo de avaliação realizado nos locais de treinamento dos atletas com horário pré-agendado com ambos os grupos. Os 
atletas foram submetidos a dois testes: Step Down Test (SDT) e Bridge Prone Test (BPT).

Para a realização do Step Down Test (SDT) e consequente avaliação do valgo dinâmico do joelho foram posicionados marcadores reflexivos em ambos os membros inferiores na correspondência anatômica dos maléolos laterais, interlinha lateral dos joelhos, e no ponto médio entre o trocânter maior e a interlinha lateral dos joelhos ${ }^{26}$, foi considerado o membro inferior que estava apoiado para análise, e solicitado que fosse realizado com ambos os membros. Os atletas foram posicionados sobre uma plataforma de 20 centímetros, foi aleatorizado o membro inferior para iniciar o teste afim de evitar influência de membro dominante ou não dominante ${ }^{25}$, e após a fase de preparação, foram instruídos a realizar 10 repetições consecutivas do movimento de descer o degrau em apoio unipodal sem retirar o calcâneo apoiado no caixote durante as repetições.

A imagem do Step Down Test foi capturada em 2D utilizando uma câmera fotográfica digital, 10 Mega Pixels, da marca Sony ${ }^{\circledR}$, localizada à frente do atleta e estabilizada por um tripé com 1,5 metros de altura e a 3 metros do local estabelecido, no plano horizontal, com uma margem de um metro de cada lado até os marcadores. As imagens foram capturadas por meio do software Virtual Dub ${ }^{\circledR 24} \mathrm{e}$ foram analisados os valores angulares para o valgo dinâmico do joelho através do software Image $\mathrm{J}^{\circledR}$, avaliado o ângulo formado entre os marcadores reflexivos.

Para o Bridge Prone Test, o atleta foi orientado a permanecer na posição de prancha ventral, antebraços e pés atuaram como pontos de apoio, colocados para coincidir com a largura do quadril, cotovelos colocados verticalmente abaixo dos ombros com os antebraços e dedos que se estenderam para a frente $e^{27,28}$. O pescoço foi mantido neutro para que o corpo permanecesse em linha reta da cabeça aos calcanhares, e a atenção foi voltada à manutenção da posição neutra das articulaçõos do quadril, pelve e coluna lombar ${ }^{27,29}$. Todos os atletas foram orientados a realizar o primeiro teste para compreensão do exercício proposto, com a finalidade de minimizar o efeito aprendizado ${ }^{29}$. Na sequência foram realizadas três repetiçôes e para o resultado utilizou-se a média do tempo de permanência em segundos entre as três tentativas. Considerou-se como termino do teste o momente em que $\mathrm{o}$ atleta não conseguisse permanecer na postura correta inicialmente orientada.

Para a análise estatística, foi realizada através do software $\operatorname{SPSS}^{\circledR} 20$, e utilizados os testes: Shapiro Wilk, Mann Whitney U e teste de Correlação de Spearmann, com nível de significância estabelecido em $5 \%$.

\section{Resultados}

A caracterização da amostra total foi não paramétrica (TABELA 1). Quando correlacionado os dois testes: SDT com BPT em relação à amostra total, a correlação foi fraca (TABELA 2). Como também se obteve o mesmo resultado quando correlacionados os testes separados nos subgrupos feminino e masculino, Rho $=0,074$ e 0,304 , respectivamente.

Ainda, quando analisados os resultados nos subgrupos, separados em função do gênero, foram
06 e 14 performances em valgo do Step Down Test inclusas, do grupo feminino e masculino respectivamente, considerado o membro inferior em apoio para análise. Todos os indivíduos apresentaram alinhamento neutro do joelho, considerado menor que $6,5^{\circ}$ para varo ou valgo ${ }^{30}$. Foi possível verificar diferença significativa quando comparado o desempenho em prancha ventral, em favor do grupo masculino (TABELA 3).

TABELA 1 -Caracterização da amostra geral e distribuída em grupos futebol masculino e futsal feminino.

\begin{tabular}{ccccc}
\hline & $\begin{array}{c}\text { Amostra total } \\
(\mathrm{N}=20) \\
\text { Mediana } \\
(\text { Min/Max })\end{array}$ & $\begin{array}{c}\text { Futebol Masculino } \\
(\mathrm{N}=14)\end{array}$ & $\begin{array}{c}\text { Futsal Feminino } \\
(\mathrm{N}=6)\end{array}$ & $\begin{array}{c}\text { Pediana (Min/Max }) \\
\text { Comparaçáo entre } \\
\text { subgrupos }\end{array}$ \\
\hline Idade (anos) & $17(13 / 20)$ & $17(15 / 19)$ & $17(13 / 20)$ & 0,407 \\
Peso (Kg) & $65,0(48,0 / 99,0)$ & $70,5(51,0 / 99,0)$ & $53,0(48,0 / 87,0)$ & $0,024^{*}$ \\
Altura (metros) & $1,75(1,55 / 1,93)$ & $1,79(1,68 / 1,93)$ & $1,65(1,55 / 1,78)$ & $0,000^{*}$ \\
IMC & $21,71(18,07 / 30,90)$ & $21,18(18,07 / 30,90)$ & $21,8(18,29 / 27,46)$ & 0,753 \\
\hline
\end{tabular}

IMC: Índice de Massa Corporal; Min: Mínimo; Max: Máximo ${ }^{*}$ Houve diferença significativa. 
Os valores referentes aos resultados do teste de correlaçáa de Spearmann (Rho).

DP: Desvio Padrão. *Diferença significativa.

TABELA 2 -Correlação entre o Step Down Test e o Bridge Prone Test.

\begin{tabular}{ccc}
\hline & Bridge Prone Test & Step Down Test \\
\hline Step Down Test (valores angulares) & 0,248 & 1,000 \\
Bridge Prone Test (segundos) & 1,000 & 0,248 \\
\hline
\end{tabular}

TABELA 3-Desempenho dos grupos masculino e feminino nos testes Step Down Test e Bridge Prone Test.

\begin{tabular}{cccc}
\hline & $\begin{array}{c}\text { Futebol Masculino } \\
(\mathrm{N}=14)\end{array}$ & $\begin{array}{c}\text { Futsal Feminino } \\
(\mathrm{N}=6)\end{array}$ & $\mathrm{P}$ \\
Mediana (Min/Max $)$ & Mediana (Min/Max) & 0,444 \\
\hline $\begin{array}{c}\text { Step Down Test } \\
\text { (valores angulares) } \\
\begin{array}{c}\text { Bridge Prone Test } \\
\text { (segundos) }\end{array}\end{array}$ & $3,00(1,00 / 13,00)$ & $3,00(1,67 / 9,33)$ & $0,000^{*}$ \\
\hline
\end{tabular}

\section{Discussão}

O objetivo deste estudo foi correlacionar a estabilidade do tronco com o valgo dinâmico do joelho em atletas, e na sequencia expandir esta análise em função do gênero. Nossos resultados apontaram fraca correlação entre estabilidade do tronco e valgo dinâmico do joelho nos atletas avaliados, assim como quando distribuídos por gênero, contrariando os relatos da literatura ${ }^{25}$.

Teoriza-se que o aumento do valgo dinâmico do joelho pode estar relacionado à fraqueza dos músculos abdutores e rotadores laterais do quadril ${ }^{31}$. No estudo de Almeida, et al. ${ }^{25}$ foi encontrada forte correlação negativa dos músculos abdutores do quadril com o APPF, em indivíduos saudáveis, ou seja, quanto maior a fraqueza destes músculos maior o ângulo encontrado no APPF. Diferente destes achados outros estudos afirmaram que a maior força dos músculos rotadores laterais e abdutores do quadril estão associados ao valgo dinâmico do joelho ${ }^{32,33}$.

Ainda, Sigward, Ota e Powers ${ }^{34}$ e Thijs, et al. ${ }^{35}$ não estabeleceram qualquer associação, como os resultados obtidos em nosso estudo. Entretanto ainda é escasso o número de estudos que avaliam a influência da resistência dos estabilizadores do quadril e do tronco no valgo dinâmico ${ }^{25}$. Nota-se que os estudos encontrados na literatura avaliem apenas a parte de resistência muscular do quadril; diferente destes, o nosso estudo traz enfoque na resistência na musculatura do complexo lombopélvico-quadril, como um todo.

A estabilização do complexo lombo-pélvicoquadril é formada por músculos estabilizadores profundos (locais) e superficiais (globais), em sua maioria tônica, conhecidos na literatura como CORE. A estabilidade do CORE é definida como a capacidade de controlar o tronco em resposta a distúrbios internos e externos, bem como perturbações esperadas ou não, que permite produção, transferência e controle de forças e movimento dos segmentos distais de uma cadeia cinética $^{36,25}$. A prancha ventral é apontada como um exercício funcional por fornecer avaliação da resistência muscular durante a ativação simultânea de toda a cadeia anterior. Testes de prancha recrutam e desafiam a musculatura anterior do CORE, ou seja, desafiam a musculatura do plano frontal contra o vetor da gravidade, especificamente, o oblíquo externo e estabilizadores laterais. $\mathrm{O}$ aumento do recrutamento e resistência dessa musculatura tem mostrado melhora da performance ${ }^{26}$. Ademais, estudos apontam que o reto abdominal e oblíquo externo são importantes para a prevenção de lesão e melhora do desempenho esportivo ${ }^{28,37,38}$.

Neste contexto, os testes de desempenho funcional têm grande importância para identificação de desequilíbrios do controle neuromuscular e têm sido utilizados para avaliar a funcionalidade, o equilíbrio dinâmico e coordenação. Sabe-se que os testes funcionais são instrumentos de medida útil, pois mensuram a função e avaliam quantitativamente os progressos na reabilitação ${ }^{39}$. Para a avaliação da capacidade de resistência dos músculos do CORE foi escolhido o exercício em "prancha ventral", por ser um exercício popular no meio futebolístico ${ }^{40}$. E SCHellendBerg, et al. ${ }^{41}$ 
sugere que o BPT é uma forma segura de avaliação de endurance abdominal, por ser um mecanismo que infere eficácia, além de ser simples de ser administrado, porque existem poucas instruçôes e maior objetividade do avaliador para definir a técnica como adequada ou inadequada.

A técnica de estabilização central prioriza a resistência com ênfase na musculatura profunda do tronco por meio de exercícios isométricos e de baixa intensidade, ou seja, é um método de fortalecimento baseado na conscientização da contração ${ }^{42}$, é utilizada como avaliação e treinamento. O presente estudo considerou o Bridge Prone Test como um teste de capacidade de resistência do core, já que analisou a capacidade de manter um determinado nível de produção de força muscular ao longo do tempo ${ }^{26}$; neste caso o tempo máximo de permanência no $\mathrm{BPT}$, considerado o tempo até a exaustão segundo CZAPROWSKI ${ }^{27}$ até que a manutenção da postura de prancha falhasse. Ainda, o estudo de STRAnd, et al. ${ }^{28}$ destaca que o motivo de finalização do teste foi a fadiga dos músculos abdominais.

Quando utilizado para avaliação, STRAND, et al. ${ }^{28}$ observou diferença na execução do teste de endurance na prancha ventral entre o grupo do sexo masculino e feminino, o primeiro grupo apresentou desempenho do teste cerca de $49 \%$ a mais que o segundo grupo, e a média encontrada nos atletas do grupo masculino foi de 110 e 74 segundos no grupo feminino. Corroborando com o estudo de SCHellenberg, et al. ${ }^{41}$ que também evidenciou diferença entres os grupos em função do gênero. Assim, o nosso estudo e os estudos de STRAND, et al. ${ }^{28}$ e SCHELLENBERG, et al. ${ }^{41}$ apontaram desempenho menor no teste para o grupo feminino.

Ainda, na literatura o estudo de SILVA ${ }^{40}$ mostrou que o exercício de exaustão em prancha ventral evidenciou diferença do tempo de execução do teste de acordo com a posição ocupada durante o jogo, atacantes e meio-campistas apresentaram $35 \%$ e $48,4 \%$ de tempo maior de execução do teste, fator que pode ser considerado limitante para o nosso estudo que não foi avaliado a interferência da posição do jogador. Ainda SILVA ${ }^{40}$ evidenciou correlação fraca entre o tempo até a exaustão e a profissionalização do atleta, ou seja, independe do seu tempo de profissionalização.

Por fim, quanto a análise do valgo dinâmico do joelho através do Step Down Test, foi analisado por imagem 2D. A literatura estabelece que o padrão ouro para análise de movimento é a técnica de imagem tridimensional (3D), método mais utilizado para analisar e quantificar o alinhamento do membro inferior em atividades com descarga de peso $^{43}$. Entretanto, este método é pouco viável para uso clinico diário e esportivo devido a seu alto custo.

Dessa forma, imagens bidimensionais (2D) necessitam de equipamentos mais simples, portáteis e com custos menores, o que facilita sua utilização e disseminação no meio clínico ${ }^{29}$, bem como no esporte. Neste sentido a análise 2D, no plano frontal, foi validada previamente com a análise $3 \mathrm{D}^{32,44}$ e tem sido utilizada em estudos com Síndrome da Dor Femoropatelar e em atletas ${ }^{33,45}$. Ainda, a utilização do método em 2D para avaliar o valgo dinâmico do joelho (ou ângulo de projeção no plano frontal), possui capacidade e confiabilidade já estabelecidas por BitTENCOURT, et al. ${ }^{14}$.

Um dos pontos estudados na literatura é o aumento do valgo do joelho e sua relação ao gênero feminino, vários estudos ${ }^{46-50}$ avaliaram o valgo do joelho e descreveram que mulheres apresentam valgismo mais acentuado do que os homens devido a sua anatomia, retardo na ativação muscular, menor rigidez articular e massa corpórea ${ }^{23}$. Assim, Russel, et al. ${ }^{48}$ demonstram maior movimento em valgo do joelho durante atividades de salto em mulheres devido as suas características. Diferente do presente estudo e do esperado, não foi encontrado diferença no valgo dinâmico do joelho entre os gêneros.

No presente estudo, a padronização da descida no SDT foi à altura de 20 centímetros da plataforma onde estavam os membros inferiores estavam apoiados, o que pode ter influenciado no ângulo de flexão do joelho apoiado em funçáo da altura e tamanho do membro inferior da voluntária, e poderia ser solucionado através do controle do ângulo de flexão do joelho em futuros estudos, para melhor precisão dos dados e padronização do recrutamento muscular. Também se sugere maior rigor com os critérios de inclusão, e outras possíveis variáveis a serem observadas é a influência da posição do jogador no campo e com o desempenho dos testes, bem como a relação do varo do joelho com a força do core. Por fim, o teste SDT é realizado no plano frontal enquanto o BPT desafia a musculatura do plano frontal, portanto, apesar das diferenças dos testes, ambos estão interligados durante a movimentação do corpo humano, entretanto ainda são necessárias novas pesquisas que avaliem a força do complexo lombo-pélvico-quadril e a sua correlação ao valgo 
dinâmico do joelho em atletas.

Conclui-se que, ao contrário do relato literatura, não houve correlação do valgo dinâmico com a resistência dos músculos do tronco, entretanto grupo masculino apresentou maior tempo de execução do teste de resistência dos músculos do CORE, através do BPT. Não foi observada diferença quando analisado o valgo dinâmico em função do gênero. Como contribuição clinica aponta-se a possibilidade de uso do BPT e do SDT como parâmetro de avaliação de atletas, realizados de forma fácil e acessível.

\section{Abstract \\ Correlation between central trunk stabilization and dynamic knee valgus in futsal and soccer athletes}

Some authors have the hipothesis about the increase of stability and neuromuscular control of lombo-pelvico-quadril complex will decrease the knee injuries, however another authors says the opposite. The aim of this study is observe correlation between central stability and dynamic knee valgus in athletes. The sample of the study was 20 athletes (six of women futsal and fourteen of men soccer team). They were randomized for two tests: Bridge Prone Test (BPT) for analyze central stability and Step Down Test (SDT) for dynamic knee valgus. For BPT performance they were oriented for stay at prone position for the longest time they could, the test was performed three times for each volunteer with one minute of rest. For the SDT the athletes were positioned on step with 20 centimeters and three reflexive points for measure de valgus angle. They were oriented to did ten repetitions of knee flexion, and was analyzed just the limb on the floor. The test was performed with both lower limbs, and was filmed and analyzed with free software Virtual Dub and ImageJ. For statistical analyses was used Shapiro Wilk test, Mann Whitney U test and Spearmann's correlation with SPSS 20.0, and it was established 5\% of significance. The result suggest was difference on BPT when analyzed with subgroups, in function of genus $(p=0,028)$. However, when correlated the both tests: BPT and SDT was observed weak correlation $(r h o=0,109)$. The same was checked when separated with genus, men's group $(\mathrm{rho}=0,279)$ and women's group $(\mathrm{rho}=0,198)$. This study shows weak correlation between central stability and dynamic knee varus in athletes, however men has a favorable significant difference with women in BPT.

\section{Referências}

1. Ribeiro CZP, Akashi PMH, Sacco ICN, Pedrinelli A. Relação entre alteraçóes posturais e lesões do aparelho locomotor em atletas de futebol de salão. Rev Bras Med Esport. 2003;9(2):91-97.

2. Keller CS, Noyes FR, Buncher R. The medical aspects of soccer injury epidemiology. Am J Sports Med. 1987;15(3):2307.

3. Fonseca ST, Ocarino JM, Silva PL. Integration of stress and their relationship to the kinetic chain. In: Magee DJ, Zachazewski JE, Quillen WS, organizadores. Science foundations and principles of pratice in musculoskeletal rehabilitation. St Louis: Saunders; 2007.

4. Heidt RS, Sweeterman LM, Richele L, Carlonas MS, Traub JA, Tekulve FX. Avoidance of soccer injuries with preseason conditioning. Am J Sports Med. 2000;28(5):659-62.

5. Hägglund M, Waldén M, Bahr R, Ekstrand J. Methods for epidemiological study of injuries to professional football players: developing the UEFA model. Br J Sports Med. 2005;39(6):340-6.

6. Triques PD. A prática precoce do futsal por crianças em situação de treinamento. Rev Saberes e Fazeres Educativos. 2005;4:33-5.

7. Rezer R, Saad MA. Futebol e Futsal: possibilidades e limitaçôes da prática pedagógica em escolinhas. Chapecó: Argos; 2005.

8. Kurata DM, Junior JM, Nowotny JP. Incidência de Lesôes em Atletas Praticantes de Futsal. Iniciação Científica CESUMAR. 2007;9:45-51 
9. Abrahão GS, Caixeta LF, Barbosa LR, Siqueira DP, Carvalho LC, Matheus JP. Incidência das lesões ortopédicas por segmento anatômico associado à avaliação da frequência e intensidade da dor em uma equipe de futebol amador. Braz J Biomotricity. 2009;3:152-8.

10. Hewett TE, Zazulak BT, Myer GD, Ford KR. A review of electromyographic activation levels, timing differences, and increased anterior cruciate ligament injury incidence in female athletes. Br J Sports Med. 2005;39(6):347-50.

11. Hewett TE, Myer GD, Ford KR, Slauterbeck JR. Dynamic neuromuscular analysis training for preventing anterior cruciate ligament injury in female athletes. Instr Course Lect. 2007;56:397-406.

12. Jacobs CC, Uhi TL, Mattacola CG, Shapiro R, Rayens WS. Hip abductor function and lower extremity landing kinematics: sex differences. J Athi Train. 2007;42(1):76-83.

13. De Aquino CF, Gonçalves GGP, da Fonseca ST, Mancini MC. Análise da relação entre flexibilidade e rigidez passiva dos isquiotibiais. Rev Bras Med Esporte. 2006;12:195-200.

14. Bittencourt NFN, Ocarino JM, Mendonça LD, Hewett TE, Fonseca ST. Foot and hip contributions to high frontal plane knee projection angle in athletes: a classification and regression tree aproach. J Orthop Sports Phys Ther. 2012;42(12):996-1004.

15. Niemuth PE, Johnson RJ, Myers MJ, Thieman TJ. Hip Muscles Weakness and Overuse Injuries in Recreational Runners. Clin J Sport Med. 2005;15:14-21.

16. McClay I, Manal K. A comparison of three-dimensional lower extremity kinematics during running between excessive pronators and normals. Clin Biomech. 1998;13(3):195-203.

17. Geiser CF, O'Connor KM, Earl JE. Effects of isolated hip abductor fatigue on frontal plane knee mechanics. Med Sci Sports Exerc. 2010;42(3):535-545.

18. Heinert BL, Kernozek TW, Grany JF, Fater DC. Hip abductor weakness and lower extremity kinematics during running. J Sports Rehabil. 2008;17(3):243-256.

19. Hall SJ. Biomecânica Básica. 4a ed. Rio de Janeiro: Guanabara Koogan; 2005.

20. Myer GD, Chu DA, Brent JL, Hewett TE. Trunk and hip control neuromuscular training for the prevention of knee joint injury. Clin Sports Med. 2008;27(3):425-48.

21. Hewett TE, Torg JS, Boden BP. Video analysis of trunk and knee motion during non-contact anterior cruciate ligament injury in female athletes: lateral trunk and knee abduction motion are combined components of the injury mechanism. Br J Sports Med. 2009;43(6):417-22. doi:10.1136/bjsm.2009.059162.

22. Cashman GE. The effect of weak hip abductors or external rotators on knee valgus kinematics in healthy subjects: a systematic review. J Sport Rehabil. 2012;21(3):273-84.

23. Leite CBS, Neto FFC. Incidências de lesôes traumato ortopédicas no futebol de campo feminino e sua relação com alterações posturais. Revista Digital- Buenos Aires. 2003;9(61).

24. Maia MS, Carandina MHF, Santos MB, Cohen M. Associação do valgo dinâmico do joelho no teste de descida de degrau com a amplitude de rotação medial do quadril. Rev Bras Med Esporte. 2012;18(3).

25. Almeida GPL, Carvalho e Silva APMC, França FJR, Magalhães MO, Burke TN, Marques AP. Ângulo-q na dor patelofemoral: relação com valgo dinâmico de joelho, torque abdutor do quadril, dor e função. Rev Bras Ortop. 2016;51(2):181-186. doi: http://dx.doi.org/10.1016/j.rbo.2015.05.003.

26. Myer GD, Ford KR, Hewett TE. New method to identify athletes at high risk of ACL injury using clinic-based measurements and freeware computer analysis. Br J Sports Med. 2011;45(4):238-44.

27. Czaprowski D. Abdominal muscle EMG-activity during bridge exercises on stable and unstable surfaces. Phys Ther Sport. 2014;15(3):162-8.

28. Strand SL, Hjelm J, Shoepo TC, Fajardo MA. Norms for an Isometric Muscle Endurance Test. J Hum Kinet. 2014;40:93102.

29. Tong TK, Wu S, Nie J. Sport-specific endurance plank test for evaluation of global core. Phys Ther Sport. 2014;15(1):5863.

30. Bendjaballah MZ, Shirazi-Adl' A, Zukor DJ. Finite element analysis of knee joint in varus-valgus. Clin Biomech. $1997 ; 12(3)$.

31. Wilson JD, Davis IS. Utility of the frontal plane projection angle in females with patellofemoral pain. J Orthop Sports Phys Ther. 2008;38(10):606-15.

32. Wilson JD, Davis IS. Lower extremity strength and mechanics during jumping in women with patellofemoral pain. J Sports Rehabil. 2009;18(1):76-90.

33. Hollman JH, Ginos BE, Kozuchowski J, Vaughn AS, Krause DA, Youdas JW. Relationships between knee valgus, hip- 
muscle strength, and hip-muscle recruitment during a single-limb step-down. J Sports Rehabil. 2009;18(1):104-17.

34. Sigward SM, Ota S, Powers CM. Predictors of frontal plane knee excursion during a drop land in young female soccer players. J Orthop Sports Pyhs Ther. 2008;38(11):661-7.

35. Thijs Y, Tiggelen DV, Willems T, Clercq DD, Witvrouw E. Relationship between hip strength and frontal plane posture of the knee during a forward lunge. Br J Sports Med. 2007;41(11):723-727.

36. Kibler WB, Press J, Sciascia A. The role of core stability in athletic function. Sports Med. 2006;36(3):189-98.

37. Nadler SF, Malanga GA, Feinberg JH, Rubanni M, Moley P, Foye P. Functional performance deficits in athletes with previous lower extremity injury. Clin J Sport Med. 2002;12:73-78.

38. Watkins RG, Uppal GS, Perry J, Pink M, Dinsay JM. Dynamic electromyographic analysis of trunk musculature in professional golfers. Am J Sport Med. 1996;24:535-538.

39. Bjôrklund K, Andersonm L, Dalen N. Validity and responsiveness of the test of athletes with knee injuries: the new criterion based functional performance test instrument. Knee Surg Sports Traumatol Arthrosc. 2009;17:435-445.

40. Silva FCA. Ativação da região do core pré treinamento priométrico em jogadores de futebol masculino do distrito federal [tese]. Goiânia: Pontíficia Universidade Católica de Goiás; 2012.

41. Schellenberg KL, Lang JM, Chan KM, Burnham RS. A clinical tool for office assessment of lumbar spine stabilization endurance: prone and supine bridge maneuvers. Am J Phys Med Rehabil. 2007;86(5):380-6.

42. Akuthota V, Nadler SF. Core strengthening. Arch Phy Med Rehabil. 2004;85(3 suppl 1):S86-92.

43. Souza RB, Powers CM. Differences in hip kinematics, muscle strength, and muscle activation between subjects with and without patellofemoral pain. J Orthop Sports Phys Ther. 2009;39(1):12-9.

44. McLEan SG, Walker K, Ford KR, Myer GD, Hewett TE, Van den Bogert AJ. Evaluation of a two dimensional analysis method ad a screening and evaluation tool for anterior cruciate ligament injury. Br J Sports Med. 2005;39(6):355-62.

45. Leetun DT, Ireland ML, Wilson JD, Ballantyne BT, Davis IM. Core stability measures as risk factors for lower extremity injury in athletes. Med Sci Sports Exerc. 2004;36(6):926-34.

46. Schmitz RJ, Shultz SJ, Nguyen AD. Dynamic valgus alignment and functional strength in males and females during maturation. J Athi Train. 2009;44:26-32.

47. Mascal CL, Landel R, Powers C. Management of patelofemoral pain targeting hip, pelvis, and trunk muscle function:2 case reports. J Orthop Sports Phys Ther. 2003;33:647-60.

48. Russel KA, Palimieri RM, Zinder SM, Ingersall CD. Sex differences in valgus knee angle during single-leg drop jump. J Athl Train. 2006;41:166-71.

49. Schmtz RJ, Ficklin TK, Shimakochi Y, Nguyen AD, Beynnan BD, Perrin DH, et al. Varus and internal-external torsional knee joint stiffness differs between sexes. Am J Sports. 2008;36:1380-8.

50. Smith RMP, McLean SG, Miller JAA, Wotys EM. Association of quadriceps and hermstrings cocontraction patters with knee Joint Loading. J Athi Tran. 2009;44(3):256-63.

ENDEREÇO DE CORRESPONDÊNCIA:

Camile Ludovico Zamboti

Av. Robert Koch, 6o - Vila Operária

Londrina - Paraná - BRASIL

CEP: $86038-440$

E-mail: camileludovico@hotmail.com;

lu_pmazzer@hotmail.com

Submissão: 28/03/2016

$1^{\text {a }}$ revisão: 04/07/2016

$2^{\mathrm{a}}$ revisão: 16/02/2017

Aceito: 09/03/2017 